\title{
A near-infrared gas sensor system based on tunable laser absorption spectroscopy and its application to $\mathrm{CH}_{4} / \mathrm{C}_{2} \mathrm{H}_{2}$ detection
}

\author{
${\text { Qixin } \mathrm{He}^{\mathrm{a}} \text {, Chuantao Zheng*a,b, Huifang Liu }}^{\mathrm{a}}$, Yiding Wang ${ }^{\mathrm{a}}$, Frank K. Tittel ${ }^{\mathrm{b}}$ \\ a State Key Laboratory on Integrated Optoelectronics, College of Electronic Science and \\ Engineering, Jilin University, 2699 Qianjin Street, Changchun 130012, P.R. China; \\ ${ }^{\mathrm{b}}$ Department of Electrical and Computer Engineering, Rice University, 6100 Main Street, Houston, \\ TX 77005, USA
}

\author{
*Corresponding author. zhengchuantao@jlu.edu.cn
}

\begin{abstract}
A near-infrared (NIR) dual-channel differential gas sensor system was experimentally demonstrated based on tunable laser absorption spectroscopy (TLAS) and wavelength modulation spectroscopy (WMS). The sensor consists of four modules, including distributed feedback (DFB) lasers for the detection of targeted gases, a custom portable DFB driver compatible for butterfly-packaged DFB lasers, a $20 \mathrm{~cm}$-long open-reflective gas-sensing probe and a custom costeffective lock-in amplifier for harmonic signal extraction. The optical and electrical modules were integrated into a standalone sensor system, which possesses advantages of user-friendly operation, good stability, small volume and low cost. With different DFB lasers, the sensor system can be used to detect different gases. Two DFB diode lasers with emission wavelengths of $1.65 \mu \mathrm{m}$ and $1.53 \mu \mathrm{m}$ were used to detect $\mathrm{CH}_{4}$ and $\mathrm{C}_{2} \mathrm{H}_{2}$, respectively. Standard $\mathrm{CH}_{4}$ and $\mathrm{C}_{2} \mathrm{H}_{2}$ samples were prepared and experiments were carried out to evaluate the performance of the two-gas TLAS sensor system. The relation between the second harmonic amplitudes (2f) and gas concentrations was obtained for the two gases by means of calibration. Both the detection error and the limit of detection (LoD) were determined experimentally. The sensor system will be useful in industrial trace gas monitoring due to its use of a low-loss optical fiber and an openreflective gas-sensing probe.
\end{abstract}

Keywords: Near-infrared diode lasers, Tunable laser absorption spectroscopy, Trace gas detection, Wavelength modulation

\section{INTRODUCTION}

Methane $\left(\mathrm{CH}_{4}\right)$ and acetylene $\left(\mathrm{C}_{2} \mathrm{H}_{2}\right)$ are widely used organic raw materials in industrial processing applications. However, both target analytes are inflammable and explosive gases. A leak of these two gases will pose a threat to industrial production safety. Furthermore, $\mathrm{CH}_{4}$ and $\mathrm{C}_{2} \mathrm{H}_{2}$ are also contributors to the greenhouse effect. Therefore, sensitive and real-time detection of $\mathrm{CH}_{4}$ and $\mathrm{C}_{2} \mathrm{H}_{2}$ is important [1-2]. Tunable laser absorption spectroscopy (TLAS) was used due to its instrumental advantages that include high detection precision and accuracy, long term stability as well as fast response [3-4].

In order to suppress noises and achieve high signal-to-noise ratio (SNR) as well as high measurement sensitivity, wavelength modulation spectroscopy (WMS) technology has been widely adopted in trace gas detection [5-6]. In WMS, the DFB diode laser's emitting wavelength is sweept across a molecular absorption line and in the meantime the diode laser is modulated by a kHz-frequency signal for the extraction of harmonic signals. In this paper, we demonstrate the detection of $\mathrm{CH}_{4}$ and $\mathrm{C}_{2} \mathrm{H}_{2}$ using the TLAS technique with two DFB diode lasers centered at $1.65 \mu \mathrm{m}$ and $1.53 \mu \mathrm{m}$ respectively. A custom-made laser temperature controller with a digital proportional integration differential (PID) algorithm is used to control the temperature of the DFB lasers [7]. Furthermore, a digital orthogonal lock-in amplifier was developed to extract the second harmonic (2f) signal from the differential absorption signal, which has a simple hardware structure was integrated into the TLAS system [8]. $\mathrm{CH}_{4}$ and $\mathrm{C}_{2} \mathrm{H}_{2}$ detection experiments were carried out.

Quantum Sensing and Nano Electronics and Photonics XIV, edited by Manijeh Razeghi, Proc. of SPIE Vol. 10111, 1011135 - @ 2017 SPIE · CCC code: $0277-786 \mathrm{X} / 17 / \$ 18$ doi: $10.1117 / 12.2250965$ 


\section{SENSOR SYSTEM CONFIGURATION AND INTEGRATION OF KEY MODULES}

\subsection{Absorption line selection of $\mathrm{CH}_{4}$ and $\mathrm{C}_{2} \mathrm{H}_{2}$}

In TLAS, either a near or mid-IR semiconductor laser source can be used. Although $\mathrm{CH}_{4}$ has a larger molecular absorption intensity in the mid-IR region $(3.31 \mu \mathrm{m})$ than that in near-IR region $(1.65 \mu \mathrm{m})$, the development of a $1.65 \mu \mathrm{m}$ DFB laser (DFBL) based TDLAS sensor system is less costly than at $3.31 \mu \mathrm{m}$. Therefore, the NIR absorption band at $\sim 1.65 \mu \mathrm{m}$ was selected. The selected absorption line for $\mathrm{C}_{2} \mathrm{H}_{2}$ is located at $1.534 \mu \mathrm{m}$.

According to the high-resolution transmission (HITRAN) 2012 molecular spectroscopic database, Fig. 1(a) depicts the absorption spectrum of $\mathrm{CH}_{4}$ molecular at $\sim 1.654 \mu \mathrm{m}$, with a line strength of $\sim 10^{-21} \mathrm{~cm}^{-1} /\left(\mathrm{molecule} \cdot \mathrm{cm}^{-2}\right)$. The absorption intensities of $\mathrm{C}_{2} \mathrm{H}_{2}, \mathrm{CO}_{2}$ and $\mathrm{H}_{2} \mathrm{O}$ are $<10^{-25} \mathrm{~cm} /$ molecule as seen in Fig. 1(b). Fig. 2(a) depicts the absorption spectrum of $\mathrm{C}_{2} \mathrm{H}_{2}$ molecular at $\sim 1.534 \mu \mathrm{m}$, with a line strength of $\sim 10^{-21} \mathrm{~cm}^{-1} /\left(\right.$ molecule $\left.\cdot \mathrm{cm}^{-2}\right)$. The absorption intensities of $\mathrm{CH}_{4}$, $\mathrm{CO}_{2}$ and $\mathrm{H}_{2} \mathrm{O}$ are at less than $10^{-25} \mathrm{~cm} /$ molecule as can be seen in Fig. 2(b).
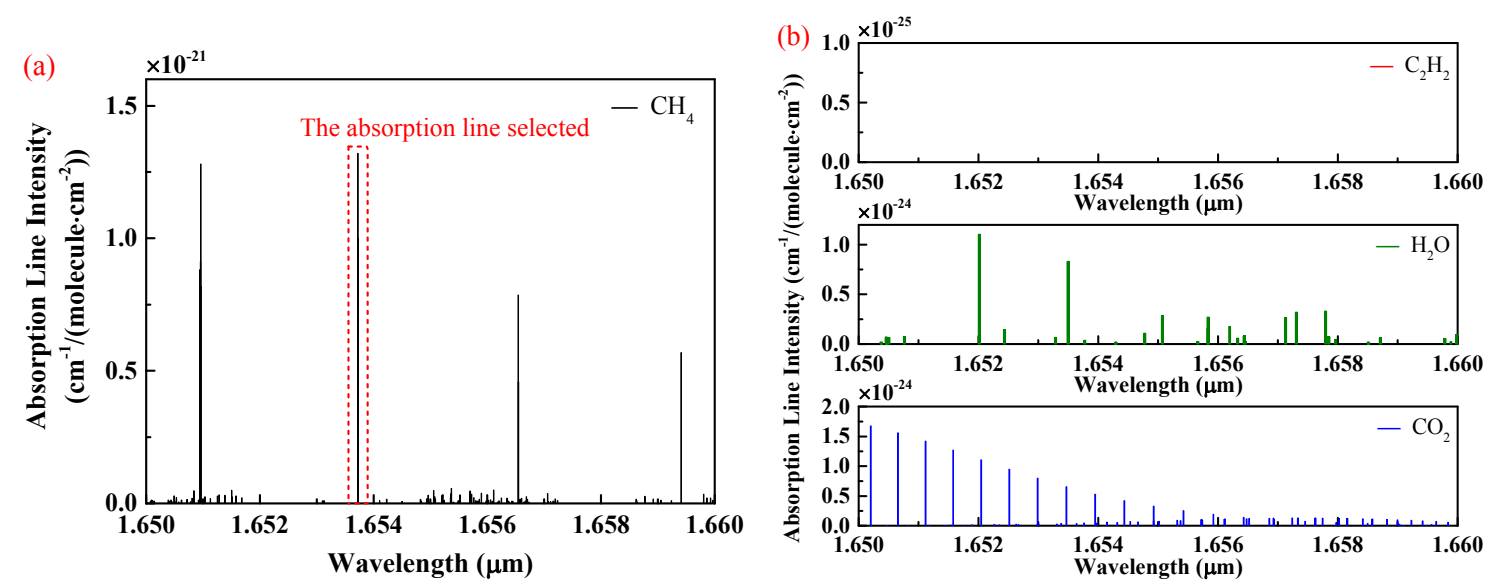

Figure 1 (a) $\mathrm{CH}_{4}$ absorption lines in the near-infrared band near $1.65 \mu \mathrm{m}$. (b) $\mathrm{C}_{2} \mathrm{H}_{2}$ (red), $\mathrm{H}_{2} \mathrm{O}$ (green) and $\mathrm{CO}_{2}$ (blue) absorption lines in the near-infrared band around $1.65 \mu \mathrm{m}$.
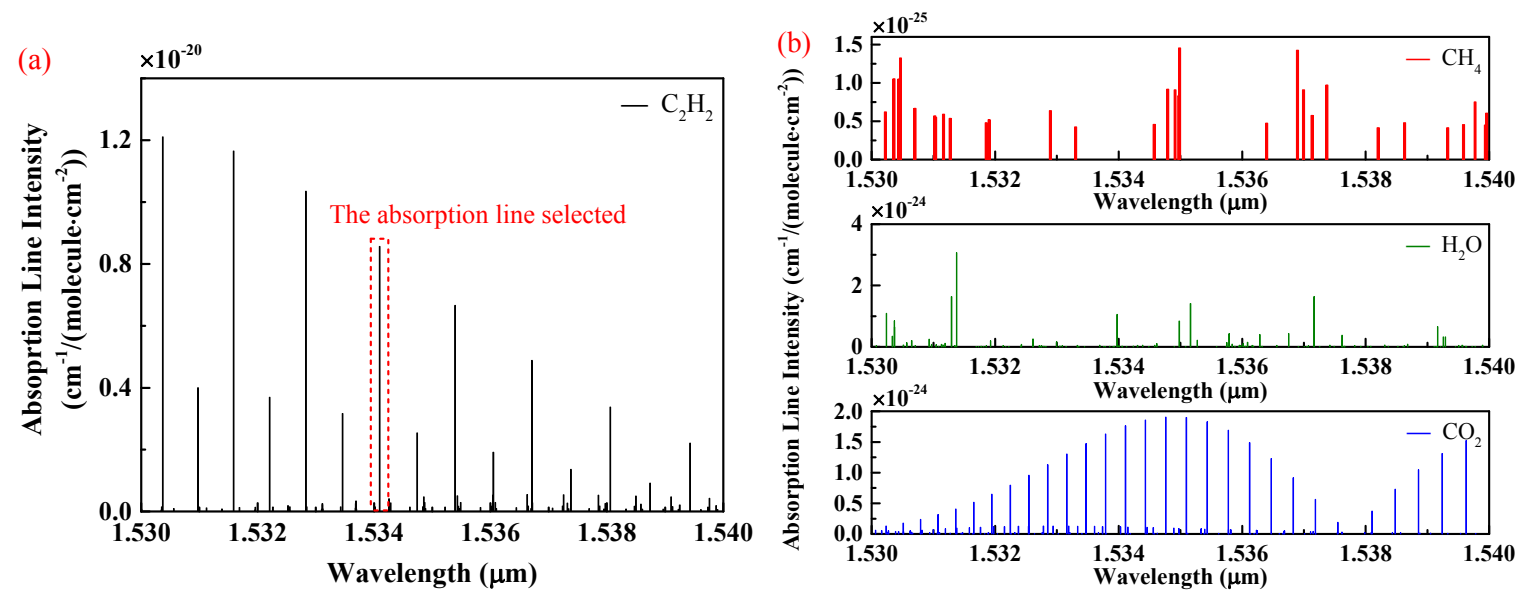

Figure 2 (a) $\mathrm{C}_{2} \mathrm{H}_{2}$ absorption lines in the near-infrared band near $1.53 \mu \mathrm{m}$. (b) $\mathrm{CH}_{4}$ (red), $\mathrm{H}_{2} \mathrm{O}$ (green) and $\mathrm{CO}_{2}$ (blue) absorption lines in the near-infrared band around $1.53 \mu \mathrm{m}$. 


\subsection{Sensor System Structure}

The layout of the NIR dual-channel differential gas sensor system is shown in Fig. 3, which consists of three modules, consisting of DFB laser driver, fiber-based optical module (including gas cell, open reflective sensing probe, fiber optical beam splitter (FOBS), fiber connectors and optical attenuator) and a data processing module.

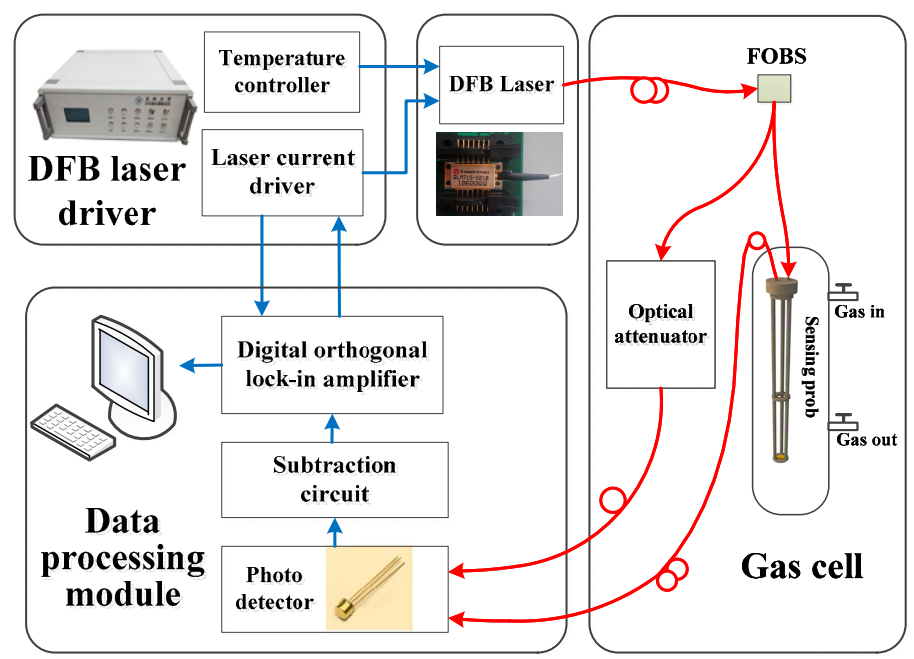

Figure 3 Schematic of the NIR dual-channel differential gas sensor system. The red lines represent single mode fibers (SMF) and the blue line represents electrical wires.

A driver board was designed, which includes a temperature control and a current modulation module. By incorporating other additional modules, such as a power supply, a liquid crystal display (LCD) and key buttons, a DFBL driver was developed, which is depicted in Fig. 4. Temperature control of the DFBL is critical for gas detection, due to the fact that the central wavelength of the diode laser depends on its operating temperature. The diode laser temperature could be varied within a range of $\pm 0.01{ }^{\circ} \mathrm{C}$ by means of a digital PID algorithm and monolithic TEC controller (Max 1968). This temperature range is small enough to avoid fluctuations of the diode laser wavelength. A saw-wave scan signal of $2 \mathrm{~Hz}$ is generated by a digital to analogue converter (DAC). Furthermore, a direct digital synthesizer (DDS, AD9851) is utilized to generate a $5 \mathrm{kHz}$ sinewave modulation signal. These two signals are combined and control the diode laser. A voltage-controlled constant current source (VCCS) module is used to convert the voltage signal into a current signal for controlling the DFBL directly.

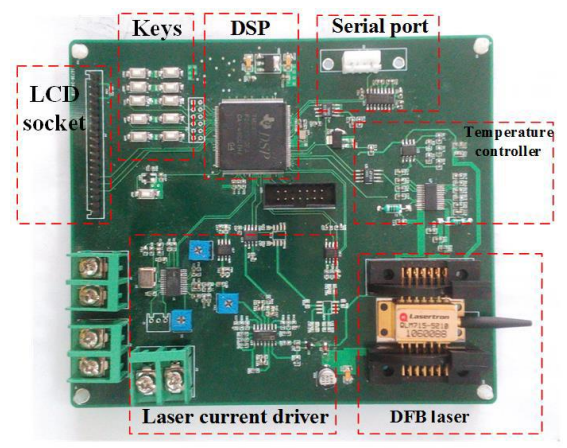

Driver Board

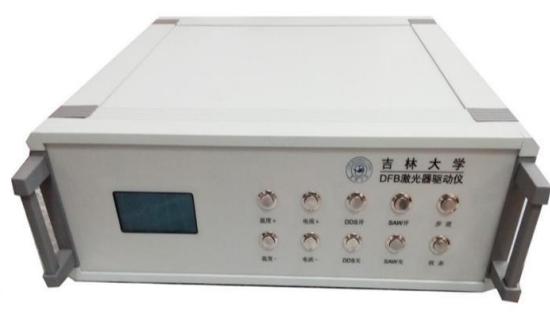

DFBL Driver

Figure 4 Left: photo of the driver board; Right: photo of the developed DFBL driver (front view).

The DFB laser output passes through the fiber optical beam splitter (FOBS) and is divided into two beams. One beam passes through an optical fiber collimator and the open reflective sensing probe in the gas cell. The other beam passes through the optical attenuator (OA) as the reference signal. The two output beams reach two InGaAs avalanche photodiode detectors (Light Sensing, model LSIAPD-50). The two signals generated by photo detectors are processed by 
a subtraction circuit, which produces a differential signal. A custom lock-in amplifier extracts a $2 f$ signal. The amplitudes of the harmonic signals are displayed by a LCD.

\section{EXPERIMENTAL RESULTS}

With two different DFBLs (both in the same 14-pin butterfly-package), the sensor system can detect different gases at $1.65 \mu \mathrm{m}$ and $1.53 \mu \mathrm{m}$ and was used to detect $\mathrm{CH}_{4}$ and $\mathrm{C}_{2} \mathrm{H}_{2}$, respectively. Standard gas samples with different concentrations were prepared to evaluate the performance of the sensor system.

\section{1 $\mathrm{CH}_{4}$ sensing performance}

For targeting the $\mathrm{CH}_{4}$ absorption line at $1.65 \mu \mathrm{m}$, the drive current of the DFB laser was set to $32 \mathrm{~mA}$, the temperature was set to $25^{\circ} \mathrm{C}$, and the pressure was $1 \mathrm{~atm}$, respectively. The scan signal was a saw-tooth signal with a frequency of 2 $\mathrm{Hz}$ and the modulation signal was a sinusoidal signal of $5 \mathrm{kHz}$.

Experiments were carried out to investigate the relationship between $\mathrm{CH}_{4}$ concentration and the amplitudes of harmonic signals. For a gas concentration range of $10^{4} \sim 5 \times 10^{4} \mathrm{ppm}$, the amplitudes of the $2 f$ harmonic signal was extracted and is depicted in Fig. 5. The $2 f$ harmonic amplitude is linear with respect to the gas concentration in agreement with TLAS theory. The plot in Fig. 5 depict that the relationship between gas concentration levels and the $2 f$ signal amplitude in the range of $10^{4} \sim 5 \times 10^{4} \mathrm{ppm}$, which can be fitted by

$$
\operatorname{Amp}\left[S_{2}(t)\right]=\left(3.970 \times 10^{-5}\right) \cdot C+1.749
$$

In order to accurately determine the LoD of the system, we measured the amplitude of the $2 f$ signal in $\mathrm{N}_{2}$ for a period of $2000 \mathrm{~s}$, as shown in Fig. 6(a). The CH4 concentration varies in the range of $-80 \sim+120$ ppm. Fig. 6(b) shows the Allan deviation plot of the detection system, where the integral time is within the range of $1 \sim 700 \mathrm{~s}$. The Allan deviation of the system is $\sim 29.52 \mathrm{ppm}$ for a 1s integration time, which is almost equal to the standard deviation (SD) of $31.2 \mathrm{ppm}$. The system shows the optimum stability with an integration time of $\sim 40 \sim 70 \mathrm{~s}$ and the corresponding Allan deviation value is $\sim 5 \mathrm{ppm}$.

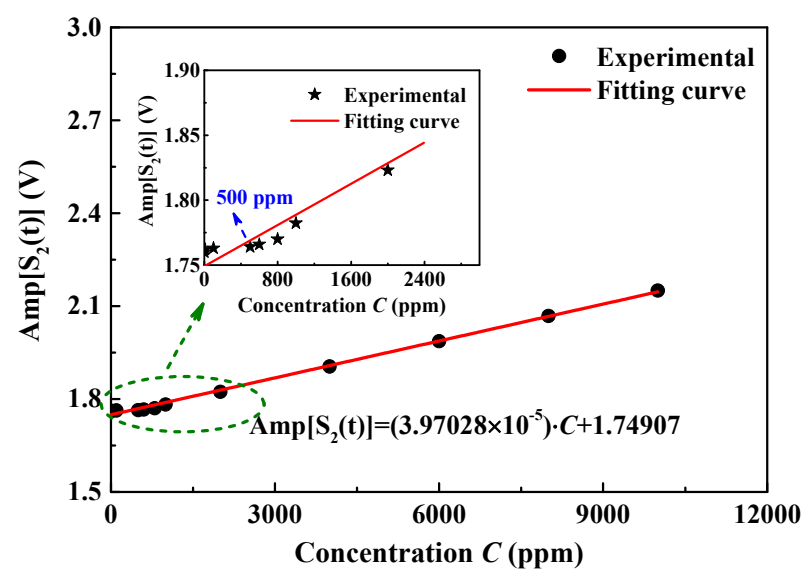

Figure $52 f$ signal's amplitude versus $\mathrm{CH}_{4}$ concentration. The inset shows the measured results of the amplitude of $2 f$ signal versus $\mathrm{CH}_{4}$ concentration near $500 \mathrm{ppm}$. 

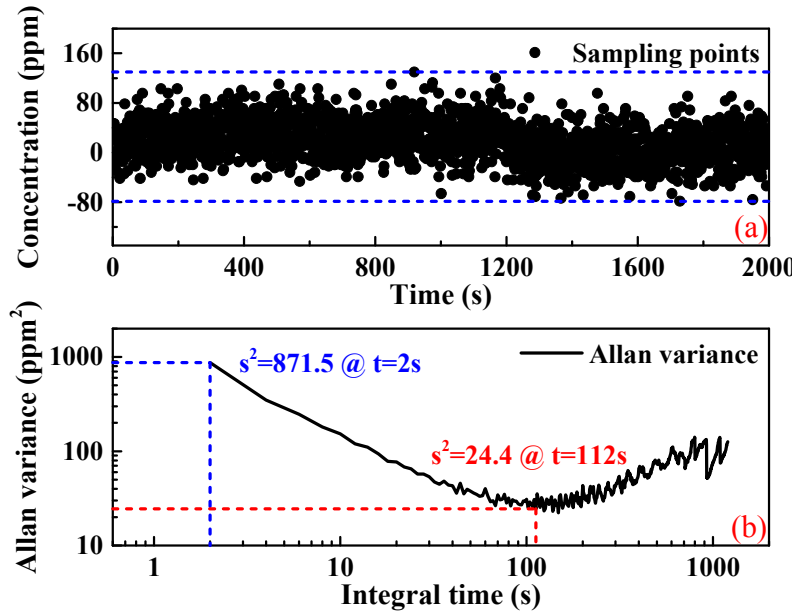

Figure 6 (a) Amplitude of the $\mathrm{CH}_{4} 2 f$ signal and concentration variation in ar $\mathrm{N}_{2}$ atmosphere for a period of $2000 \mathrm{~s}$. (b) Allan deviation plot of the $\mathrm{CH}_{4}$ detection system.

\section{2 $\mathrm{C}_{2} \mathrm{H}_{2}$ sensing performance}

For targeting the $\mathrm{C}_{2} \mathrm{H}_{2}$ absorption line at $1.53 \mu \mathrm{m}$, the drive current of the DFB laser was set to $60 \mathrm{~mA}$ and the temperature was set to $28{ }^{\circ} \mathrm{C} . \mathrm{C}_{2} \mathrm{H}_{2}$ samples within a concentration range of $0 \sim 10000 \mathrm{ppm}$ were prepared for injection into the $600 \mathrm{~mL}$ gas cell. A calibration experiment was carried out to measure the relationship between the $2 f$ signal's amplitude and the $\mathrm{C}_{2} \mathrm{H}_{2}$ concentration. The result is shown in Fig. 7. A linear relationship is observed between $\mathrm{C}_{2} \mathrm{H}_{2}$ concentration and the $2 f$ signal amplitude. The obtained fitting equation is

$$
\operatorname{Amp}\left[S_{2}(t)\right]=\left(2.135 \times 10^{-4}\right) \cdot C+0.516
$$

The correlation coefficient of the curve is 0.998223 , for the $2 f$ signal amplitude and the $\mathrm{C}_{2} \mathrm{H}_{2}$ concentration. A standard $\mathrm{C}_{2} \mathrm{H}_{2}$ gas sample with concentration of $0 \mathrm{ppm}$ was measured continuously for 2 hours at a sampling frequency of $1 \mathrm{~Hz}$. The calculated Allan variance is shown in Fig. 8(b). When the integration time is $1 \mathrm{~s}$, the Allan variance is $75.5 \mathrm{ppm}^{2}$ and the MDL of this system is $8.69 \mathrm{ppm}$. When the integration time is $10 \mathrm{~s}$, the system shows the best stability and the corresponding Allan deviation value is $\sim 5 \mathrm{ppm}$.

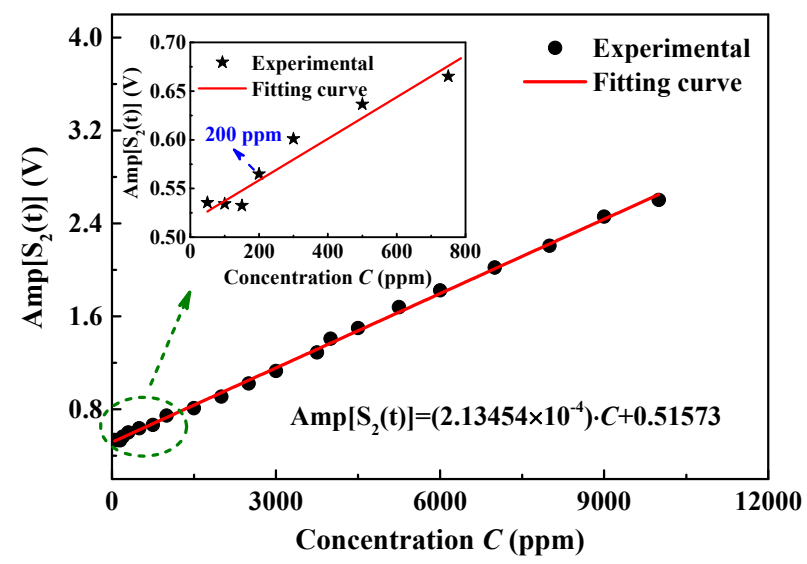

Figure $72 f$ signal's amplitude versus $\mathrm{C}_{2} \mathrm{H}_{2}$ concentration. The inset shows the measured results of the the $2 f$ amplitude as a function of the $\mathrm{C}_{2} \mathrm{H}_{2}$ concentration at $\sim 200 \mathrm{ppm}$. 

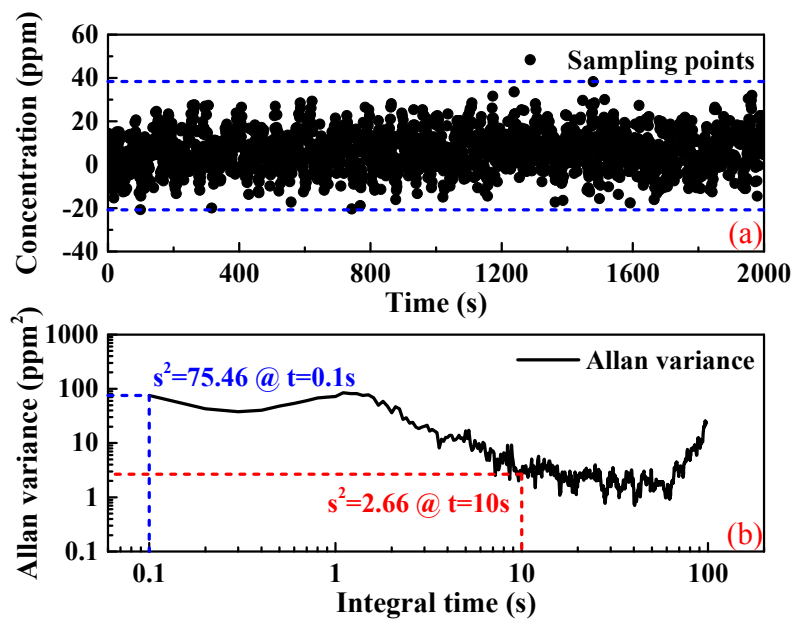

Figure 8 (a) The amplitude of the $2 f$ signal and concentration variation in a $\mathrm{N}_{2}$ atmosphere for a period of $2000 \mathrm{~s}$. (b) Allan deviation curve of the detection system.

\section{CONCLUSION}

A near-infrared (NIR) dual-channel differential gas sensor system based on the TLAS and WMS technique, which consists of a DFBL driver module, an open reflective gas sensing probe inside a gas cell, an InGaAs photodiode and data processing module. Both a digital PID temperature controller and a wavelength modulation module were developed to operate the DFB laser and a digital orthogonal lock-in amplifier to extract the $2 f$ signals. Diode laser temperature fluctuations could be limited within the range of $-0.01 \sim 0.01{ }^{\circ} \mathrm{C}$ by both temperature and injection current control. $\mathrm{CH}_{4}$ and $\mathrm{C}_{2} \mathrm{H}_{2}$ detection experiments were carried out to investigate the system performance. For $\mathrm{CH}_{4}$ detection, the amplitudes of the $2 f$ harmonic signal was obtained as the $\mathrm{CH}_{4}$ concentration increases from 0 to $5 \times 10^{4} \mathrm{ppm}$. The LoD for $\mathrm{CH}_{4}$ was $29.52 \mathrm{ppm}$ for an effective a path length of $40 \mathrm{~cm}$, indicating a minimum detectable sensitivity of $12 \mathrm{ppm} \bullet \mathrm{m}$. For $\mathrm{C}_{2} \mathrm{H}_{2}$ detection, the experimental results indicate that the system has also good linearity and stability. Based on the Allan deviation at an integral time of $1 \mathrm{~s}$, the LoD was $8.69 \mathrm{ppm}$, indicating a minimum detectable column density of 3.5 $\mathrm{ppm} \bullet \mathrm{m}$. The reported sensor system can be used for remote monitoring of industrial processes.

\section{ACKNOWLEDGEMENTS}

Q. He, C. Zheng, H. Liu and Y. Wang wish to express their gratitude to the National Natural Science Foundation of China (Nos. 61307124 and 61627823), the National Key Technology R\&D Program of China (Nos. 2013BAK06B04 and 2014BAD08B03), the Science and Technology Department of Jilin Province of China (Nos. 20120707 and 20140307014SF), the Education Department of Jilin Province of China, the China Scholar Council Supported Program (No. 201606170210, the Changchun Municipal Science and Technology Bureau (Nos. 11GH01 and 14KG022), and the State Key Laboratory of Integrated Optoelectronics, Jilin University (no. IOSKL2012ZZ12) for their generous support of this work. F.K.Tittel acknowledges financial support of a research grant \# C-0586 from the Welch Foundation.

\section{REFERENCES}

[1] Durry, G., Lij, S., Vinogradov, I., Titov,A., Joly,L., Cousin, J., Decarpenterie,T., Amarouche, N., Liu, X., Parvitte, B., Korablev, O., Gerasimov, M. and Zéninari, V., "Near infrared diode laser spectroscopy of C2H2, H2O, CO2 and their isotopologues and the application to TDLAS, a tunable diode laser spectrometer for the martian PHOBOS-GRUNT space mission", Applied Physics B: Lasers and Optics Papers 99(1), 339-351 (2010).

[2] Ye, W.L., Zheng, C.T., Yu, X., Zhao, C.X., Song, Z.W. and Wang, Y.D., "Design and performances of a midinfrared CH4 detection device with novel three-channel-based LS-FTF self-adaptive denoising structure", Sensors and Actuators B: Chemical Papers 155(1), 37-35 (2011) 
[3] Zheng, C.T., Ye, W.L., Huang, J.Q., Cao, T.S., Lv, M., Dang, J.M., Wang, Y.D., "Performance improvement of a near-infrared $\mathrm{CH} 4$ detection device using wavelet-denoising-assisted wavelength modulation technique", Sensors and Actuators B: Chemical Papers 190, 249-258 (2014)

[4] Utsav, K.C., Nasir, F.E., Aamir, F., "A mid-infrared absorption diagnostic for acetylene detection", Applied Physics B Papers 120(2), 223-232 (2015).

[5] Cao, Y.C., Jin, W., Yang, F., Ho, H.L., "Ultra-sensitive all-fibre photothermal spectroscopy with large dynamic range", Optics Express Papers 22, 13190-13201 (2014).

[6] Wei, W., Chang, J., Huang, Q., Zhu, C., Wang, Q., Wang,Z., Lv, G., "Wavelength modulation spectroscopy with signal-reference beam method for highly sensitive gas detection", Applied Physics B: Lasers and Optics Papers 118(1) 75-83 (2015).

[7] Shemshad, J., Aminossadati, S.M., Kizil, M.S., "A review of developments in near infrared methane detection based on tunable diode laser", Sensors and Actuators B: Chemical Papers 171(8) 77-92 (2012).

[8] Gao, Q., Zhang, Y., Yu, J., Wu, S., Zhang, Z., Zheng, F., Lou, X., Guo, W., "Tunable multi-mode diode laser absorption spectroscopy for methane detection", Sensors and Actuators A: Physical Papers 199(9) 106-110 (2013). 